\title{
Analysis of the Clinical Value of Exhaled Nitric Oxide in Bronchial Asthma with Allergic Rhinitis
}

\author{
Jing Sun ${ }^{1}$, Weijun Cao ${ }^{2}$, Xianying Gai ${ }^{1}$, Xiaofang Wang ${ }^{1}$ \\ ${ }^{1}$ Sijing Hospital of Shanghai Songjiang District, Songjiang, Shanghai, 201601 \\ ${ }^{2}$ Shanghai Lung Hospital, Yangpu, Shanghai, 201433
}

Keywords: Exhalation of Nitric Oxide, Allergic Rhinitis, Bronchial Asthma, Percentage of Eosinophils in Peripheral Blood, Serum Total IgE, Pulmonary Function

\begin{abstract}
The paper makes a comparative analysis of the clinical application value of exhaled nitric oxide (FeNO) in bronchial asthma combined with allergic rhinitis and simple bronchial asthma. It includes the comparison of the difference in FeNO measurement value, the correlation between FeNO and peripheral blood eosinophil percentage (EOS\%), serum total specific antibody IgE (IgE) and lung function. In the paper, a total of 53 patients diagnosed and treated in Sijing Hospital of Shanghai Songjiang District were collected. The following groups were as follows: bronchial asthma complicated with allergic rhinitis group (AS+AR), 18 patients, simple bronchial asthma group (AS) 16 people, and control group 19 people. FeNO, peripheral blood EOS\%, serum total IgE and lung function were measured respectively. Through SPSS statistical software analysis, it is concluded that there is a significant difference in terms of FeNO among AS+AR group, AS group and control group. The difference between AS+AR group and AS group is statistically significant $(\mathrm{P}<0.001)$, compared with those of AS group (three): the AS+AR group is higher than that of AS and control group, and the AS group is higher than that of the control group (22). There was a significant difference in EOS\% between AS+AR group and AS group $(\mathrm{P}<0.001)$. There was a great difference between AS+AR group and control group $(\mathrm{P}<0.001)$. There was no significant difference between AS group and control group $(\mathrm{P}=0.102)$. The mean value of $\mathrm{AS}+\mathrm{AR}$ group was higher than that of AS group and control group, and that of AS group was higher than that of control group. The total IgE was significantly different between the AS+AR group and the control group $(\mathrm{P}<0.01)$. There was a significant difference between the AS group and the control group $(\mathrm{P}<0.001)$. There was no significant difference between the AS+AR group and the AS group $(\mathrm{P}=0.751)$, the mean value: AS+AR group was higher than that of the AS group and the control group, and the AS group was higher than the control group. There was a significant difference between FEVl and FVC\% in AS group and control group $(\mathrm{P}<0.01)$. There was a significant difference between $A S+A R$ group and control group $(\mathrm{P}<0.05)$. There was no significant difference between AS group and AS+AR group $(\mathrm{P}=0.318)$. Mean: the control group was higher than that of AS+AR group and AS group, and the AS+AR group was higher than that of group AS. Index correlation: there is a significant positive correlation between FeNO and IgE $(\mathrm{P}<0.01)$; FeNO has a significant positive correlation with Eos\% $(\mathrm{P}<0.01)$; FeNO has a significant negative correlation with FEV1/FVC\% $(\mathrm{P}<0.01)$; there is a significant positive correlation between IgE and Eos\% (IgE). Thus, FeNO is an objective index reflecting the chronic airway inflammation, objectively reflecting the degree of allergic airway inflammation, in time to assess the condition of patients with asthma, which is worthy of attention on allergic rhinitis; FeNO and EOS\%, IgE, FEVl / FVC\% complement each other, mutual influence, clinical the reference of diagnosis and treatment evaluation for allergic respiratory disease.
\end{abstract}

\section{Introduction}

Asthma is a heterogeneous disease characterized by chronic airway inflammation. It includes respiratory symptoms such as wheezing, shortness of breath, chest tightness and cough, which are changing and aggravating over time, with variable expiratory flow limitation. Asthma is often associated with direct or indirect stimulations of airway hyperresponsiveness accompanied by 
chronic airway inflammation [1-2]. Bronchial asthma complicated with allergic rhinitis is a simultaneous clinical and subclinical respiratory allergy and allergic symptoms of the upper respiratory tract. They often coexist in the same patient and interact with each other. Bronchial asthma complicated with allergic rhinitis on lower respiratory tract pathological changes were allergic inflammation of bronchial and nasal, nasal and bronchial inflammation in the pathogenesis of inducement, genetic change and local pathological changes, immune dysfunction and pathogenesis and other aspects are very similar, are based on the pathology of respiratory tract eosinophilic granulocytes increased allergic inflammation characterized by. Recommended an airway, a disease, research data show that patients with allergic rhinitis airway inflammation index of less than $68.3 \%$, bronchial asthma patients with nasal inflammatory index anomalies of about 89\%, allergic rhinitis can be increased by about 3 times Incidence [3-4]. Asthma is a reversible expiratory flow limitation is characterized for asthma, pulmonary function changes in the same patient between occlusion completely normal and severe bronchial asthma, recurrent seizures can be gradually increased airway obstruction and airway remodeling, which leads to the decrease of lung function. Exhaled nitric oxide (FeNO) has high specificity and sensitivity in asthma diagnosis and airway inflammation monitoring, and it can also reflect the allergic degree of organism to some extent. Allergy clinic laboratory tests used a total serum specific antibody (IgE) and peripheral blood eosinophils (EOS), serum IgE antibody mainly exists in individuals with allergies, these individuals in contact with specific allergens after the IgE generation a series of allergic reactions. Peripheral blood EOS is the main effector of allergic reaction. Activated EOS can release various inflammatory mediators, and play an important role in airway inflammation, development and mucosal damage [5]. The purpose of this study is to analyze the correlation between FeNO numerical difference and the correlation between FeNO and IgE, EOS\% and lung function, and to analyze the influence of allergic rhinitis on asthma management in bronchial asthma patients, so as to provide clinical reference for diagnosis and evaluation of allergic respiratory diseases.

\section{Information and Methods}

\subsection{The research object}

53 cases from October 2016 --2017 year in October in our hospital patients, including 25 cases of male, female 28 cases, average age $(57.2+3.8)$ years old, according to whether the bronchial asthma complicated with allergic rhinitis and is divided into 3 groups: bronchial asthma complicated with allergic rhinitis group (AS+ AR) in 18 cases [6]. Bronchial asthma group (AS) 16 cases control group 19 cases.

Inclusion criteria: (1) with the 2017 edition of the global initiative for asthma (GINA2017) patients (2) in accordance with the latest revision of the 2015 edition of guidelines for the diagnosis and treatment of allergic rhinitis patients (3) with good compliance, can be exhaled nitric oxide and pulmonary function test

Exclusion criteria: (1) with chronic obstructive pulmonary disease, interstitial lung disease, bronchiectasis, lung cancer and other lung diseases (2) patients suffering from parasitic infection or other activities without the control of infection, tumor, liver disease or blood disease (3) not with exhaled nitric oxide and pulmonary function test [7-8].

This study is in line with the ethical standards established by the medical ethics committee of the hospital, and is approved and obtained the informed consent of the patients.

\subsection{Research method}

\subsubsection{FeNO determination}

The determination of the system were detected by western Sweden's Karolinska limited NIOX VERO, before the test 1 hours of fasting, ban alcohol and tobacco, no movement before detection were introduced to interface detection steps and key points of detection using computer, in strict accordance with the determination of system specification and operation guide. The patient took the seat, did not hold the nose, and slowly inhaled to the total lung after calm and exhaled [9-10]. Then 
the lips were wrapped tightly by the filter to keep the airflow exhaled, and the airflow speed was no less than $50 \mathrm{ml} / \mathrm{s}$, and the expiration time was no less than 5S. After $90 \mathrm{~s}$, the system automatically displayed the FeNO number.

\subsubsection{Pulmonary function measurement}

Check the Japanese Chest-8800 pulmonary function test system test, the subjects were not seat, backrest, nose clip, including cylinder, the maximum inspiratory breath hold, to maximum power, the fastest speed until completely "emptying" expiratory lung, repeat, and then relax, no need to breath air can be exhaled, at least for 6 seconds or more [11]. FVC and FEV1 take the maximum value should be 3 times the ideal curve, this is the 3 time the ideal curve in the FVC and FEV1 value difference should not exceed $5 \%$ or $150 \mathrm{ml}$ (both must satisfy), FEV1/FVC value should be used when the maximum FEV1 and FVC values in the ideal curve (from different curve).

\subsubsection{Detection of peripheral eosinophils (EOS) in peripheral blood}

The venous blood was collected for $2 \mathrm{~mL}$, reversed and mixed for 5 minutes, and put into the snowball tester. The eosinophils count and percentage of peripheral blood were measured by the Japanese automatic five classification blood cell analyzer [12].

\subsubsection{Total IgE (TIgE) detection}

The venous blood $5 \mathrm{~mL}$ was collected, the vacuum anticoagulant tube containing the separated glue was placed, and $3000 \mathrm{r} / \mathrm{min}$ centrifugation was $10 \mathrm{~min}$, and the serum was separated. The detection of serum TIgE was carried out by the automatic operation instrument of European Mongolian blot. The clinical reference value of TIgE concentration was $<100 \mathrm{IU} / \mathrm{mL}$.

\subsection{Statistical method}

The data were analyzed by SPSS19.0. The data are expressed by ' $x+s$, differences between groups were compared using single factor analysis of variance, correlation analysis by Pearson correlation analysis, $\mathrm{P}<0.05$ for the difference was statistically significant.

\section{Results}

The values of FeNO were significantly different between AS+AR group, AS group and control group $(\mathrm{P}<0.001)$. The difference between $\mathrm{AS}+\mathrm{AR}$ group and AS group was statistically significant (22), the difference between them was statistically significant $(\mathrm{P}<0.001)$, the mean value: AS+AR group was higher than that of AS group and control group, and AS group was higher than that of control group (three).

There was a significant difference in the EOS\% value between AS+AR group and AS group $(\mathrm{P}<0.001)$. There was a significant difference between the As+AR group and the control group $(\mathrm{P}<0.001)$. There was no significant difference between the AS group and the control group $(\mathrm{P}=0.102)$, the mean value: AS+AR group was higher than that of the AS group and the control group, and the AS group was higher than the control group.

The total serum IgE was significantly different between the AS+AR group and the control group $(\mathrm{P}<0.01)$. There was a significant difference between the AS group and the control group $(\mathrm{P}<0.001)$. There was no significant difference between the AS+AR group and the AS group $(\mathrm{P}=0.751)$, the mean value: AS+AR group was higher than that of the AS group and the control group, and the AS group was higher than the control group.

There was a significant difference in FEV1/FVC\% between AS group and control group $(\mathrm{P}<0.01)$. There was a significant difference between $\mathrm{AS}+\mathrm{AR}$ group and control group $(\mathrm{P}<0.05)$, and there was no significant difference between AS group and AS+AR group $(\mathrm{P}=0.318)$. In the control group, the control group was higher than the ARAS group and the AS group, and the group ARAS was higher than the group AS. 
Table. 1

\begin{tabular}{ccc}
\hline & $\mathrm{n}$ & $-\mathrm{x} \pm \mathrm{s}$ \\
\hline FeNO(ppb) & 53 & $48.39 \pm 30.219$ \\
\hline EOS\% & 47 & $3.1021 \pm 2.76529$ \\
\hline FEV1/FVC & 35 & $78.9017 \pm 7.82706$ \\
\hline IgE $(\mathrm{u} / \mathrm{ml})$ & 38 & $116.22632 \pm 119.433373$ \\
\hline
\end{tabular}

Table.2

\begin{tabular}{|c|c|c|c|c|}
\hline & & $\mathrm{AS}+\mathrm{AR}$ & AS & Control group \\
\hline & AS+AR & & .000 & .000 \\
\hline \multirow[t]{3}{*}{ FeNO (ppb) } & $\overline{\mathrm{AS}}$ & .000 & & .000 \\
\hline & Control group & .000 & .000 & \\
\hline & AS+AR & & .000 & .000 \\
\hline \multirow[t]{3}{*}{ EOS\% } & AS & .000 & & .102 \\
\hline & Control group & .000 & .102 & \\
\hline & $\mathrm{AS}+\mathrm{AR}$ & & .318 & .018 \\
\hline \multirow[t]{3}{*}{ FEV1/FVC\% } & $\mathrm{AS}$ & .318 & & .001 \\
\hline & Control group & .018 & .001 & \\
\hline & $\mathrm{AS}+\mathrm{AR}$ & & .751 & .001 \\
\hline \multirow[t]{2}{*}{ IgE (IU/ml) } & $\overline{\mathrm{AS}}$ & .751 & & .000 \\
\hline & Control group & .001 & .000 & \\
\hline
\end{tabular}

*. The significant level of mean difference was 0.05 .

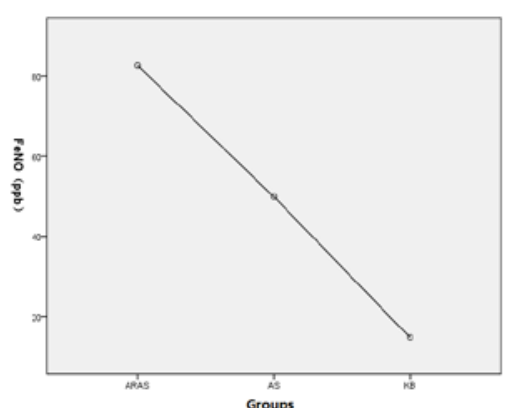

(a)

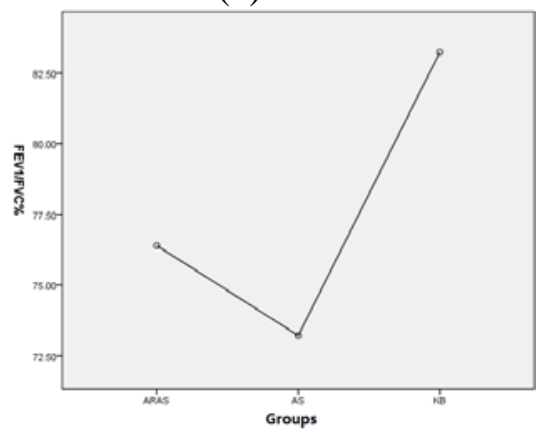

(b)

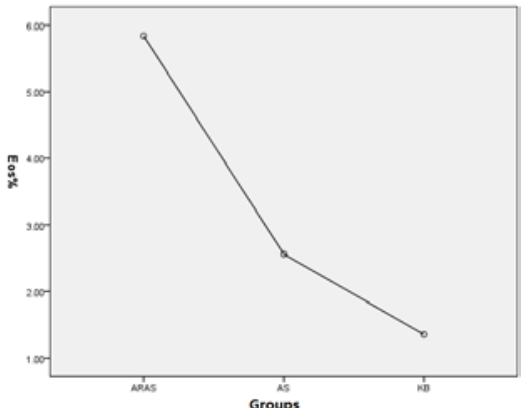

(b)

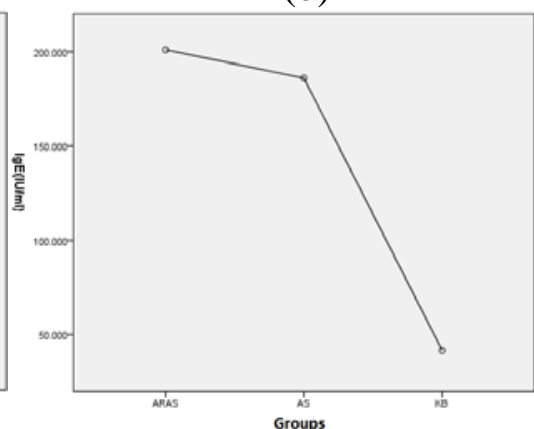

(d)

Figure.1

There was a significant positive correlation between FeNO and $\operatorname{IgE}(\mathrm{P}<0.01)$, and there was a significant positive correlation between FeNO and Eos\% $(\mathrm{P}<0.01)$.

There was a significant negative correlation between FeNO and FEV1/FVC\% $(\mathrm{P}<0.01)$, and there was a significant positive correlation between IgE and Eos\% $(\mathrm{P}<0.05)$.

The correlation between IgE and FEV1/FVC\% was not obvious ( $\mathrm{P}=0.592)$, and the correlation between Eos\% and FEV1/FVC\% was not obvious ( $\mathrm{P}=0.119)$. 
Table.3

\begin{tabular}{cccccc}
\hline & & $\begin{array}{c}\text { FeNO } \\
(\mathrm{ppb})\end{array}$ & IgE(IU/ml) & EOS\% & $\begin{array}{c}\text { FEV1/FVC } \\
\%\end{array}$ \\
\hline FeNO(ppb) & Pearson relevance & 1 & $.572^{* *}$ & $.55^{* *}$ & $-.435^{* *}$ \\
& Significance (bilateral) & & .000 & .000 & .009 \\
& $\mathrm{~N}$ & 53 & 38 & 47 & 35 \\
\hline IgE(u/ml) & Pearson relevance & $.572^{* *}$ & 1 & $.404^{*}$ & -.108 \\
& Significance (bilateral) & .000 & & .016 & .592 \\
& $\mathrm{~N}$ & 38 & 38 & 35 & 27 \\
\hline EOS\% & Pearson relevance & $.550^{* *}$ & $.404^{*}$ & 1 & -.282 \\
& Significance (bilateral) & .000 & .016 & & .119 \\
& $\mathrm{~N}$ & 47 & 35 & 47 & 32 \\
\hline FEV1/FVC\% & Pearson relevance & $-.435^{* *}$ & -.108 & -.282 & 1 \\
& Significance (bilateral) & .009 & .592 & .119 & \\
& $\mathrm{~N}$ & 35 & 27 & 32 & 35 \\
\hline
\end{tabular}

**. There is a significant correlation between the.01 level (bilateral).

*. There is a significant correlation at the 0.05 level (bilateral).

\section{Conclusions}

In this study, FeNO, EOS\% and TIgE of bronchial asthma patients with allergic rhinitis were all higher than those of patients with simple bronchial asthma. The lung function of the two groups decreased to varying degrees compared with the control group. Therefore, the possibility of anaphylactic rhinitis should be considered when the patients with bronchial asthma are found to have relatively high FeNO values during the clinical process. In a foreign city non asthma experiment, exhaled nitric oxide with atopy was higher than that in non-atopic group, also allergic rhinitis population exhaled nitric oxide was higher than that in non-allergic rhinitis group, this shows that atopic and allergic rhinitis can influence the concentration of gas called nitric oxide. Clinically, when asthma management is not effective, it is also necessary to consider the presence of allergic rhinitis. Based on the severity of allergic rhinitis, we need to consider whether we should treat allergic rhinitis at the same time. Similarly, when patients with asthma have higher EOS\% and TIgE, they should also consider the effect of allergic rhinitis. But in patients with allergic rhinitis have a higher FeNO value is necessarily associated with asthma, this remains controversial, after the current FeNO is not the gold standard for the diagnosis of asthma, but high concentration of exhaled nitric oxide in asthma has been detected, the high level of FeNO is strongly suggestive of asthma. Also found in this study, there is a positive correlation between FeNO level and peripheral blood EOS\%, TIgE, which is consistent with research results at home and abroad, FeNO and EOS on airway inflammation, can better reflect the airway eosinophilic inflammation level, and can monitor the glucocorticoid treatment effect. To a certain extent, but also reflect the specific parameters. Peripheral blood EOS also has a high diagnostic value in the diagnosis of EOS phenotype in asthma, suggesting an acute exacerbation of EOS-counted asthma, drug and immune asthma intervention compared with EOS\% and serum TIgE and FeNO levels in a significantly positive EOS-related study. This study also confirmed that there was a significant positive correlation of FeNO level and total serum IgE levels are also associated with the pathogenesis of asthma, and airway mucous layer of B lymphocytes produced by allergen specific IgE and mast cell surface IgE high affinity receptor -Fc epsilon RI and eosinophilic granulocyte cell surface the low affinity receptor -Fc epsilon RII specificity, mast cells, activate the respiratory mucosa of eosinophilic cells, induce degranulation and release activity of inflammatory mediators caused by bronchial spasm, vascular congestion and permeability enhancement, tissue inflammation, cause asthma reaction, therefore, for example when did not carry out the detection of FeNO, TIgE the level of eosinophilic inflammation of the airways also suggest a certain role. As we all know, bronchial asthma is a chronic airway inflammatory disease involving many cell and cell 
components. It is a specific chronic airway inflammation. Asthma is characterized by a reversible expiratory flow. The clinical symptoms and signs can not fully reflect the degree of inflammation. Pulmonary function monitoring can reflect the pathological changes of asthma, helps to confirm the diagnosis of asthma, asthma diagnosis, not standardized treatment, asthma management oversight, these will lead to the recurrent attacks of asthma, and gradually aggravate airway obstruction and airway remodeling, which leads to the decrease of lung function. In this study, there is a significant negative correlation between FeNO level and FEV1/FVC\% $(\mathrm{P}<0.01)$. Therefore, when patients fail to perform lung function tests, a certain level of FeNO can also play a role in indicating the degree of lung function.

There are simple, safe, noninvasive, quantitative and reproducible characteristics in the detection of common chronic airway inflammation, making it widely used in clinical evaluation of airway inflammation. Through the above discussion, when bronchial asthma in patients with high level of FeNO value need to consider whether there is effect of allergic rhinitis on it, FeNO has certain implications for bronchial asthma complicated with allergic rhinitis, FeNO and EOS\%, TIgE, FEVl / FVC\% are interrelated and influence each other in allergic airway the disease in their plays an important and complementary role for clinical diagnosis and treatment activities provide important reference.

\section{References}

[1] Huang D L Z. Clinical Analysis of Fractional Exhaled and Nasal Nitric Oxide in Allergic Rhinitis Children [J]. 2015, 06(1).

[2] Di C G, Marcucci F, Palomba A, et al. Exhaled nitric oxide in children with allergic rhinitis: a potential biomarker of asthma development. [J]. Pediatric Allergy \& Immunology, 2015, 26(1):85-87.

[3] Cara G D, Marcucci F, Palomba A, et al. Exhaled nitric oxide in children with allergic rhinitis: A potential biomarker of asthma development[M]// Pediatric Allergy and Immunology. 2015:85-87.

[4] Duongquy S, Vuminh T, Huahuy T, et al. Study of nasal exhaled nitric oxide levels in diagnosis of allergic rhinitis in subjects with and without asthma[J]. Journal of Asthma \& Allergy, 2017, 10:75-82.

[5] Moon S H, Jang H J, Park Y S, et al. Fractional exhaled nitric oxide in Korean children with allergic rhinitis $[\mathrm{J}] . \quad 2015,3(6): 439$.

[6] Park J, Lee E, Yang S I, et al. Exhaled nitric oxide and bronchial hyperresponsiveness in atopic asthmatic children with and without allergic rhinitis[J]. 2015, 3(6):425.

[7] Ciprandi G, Gallo F, Ricciardolo F L, et al. Fractional Exhaled Nitric Oxide: A Potential Biomarker in Allergic Rhinitis? [J]. International Archives of Allergy \& Immunology, 2017, 172(2):99.

[8] Tang W, Zhou J, Miao L, et al. Clinical features in patients of cough variant asthma with normal and high level of exhaled fractional nitric oxide [J]. Clinical Respiratory Journal, 2016.

[9] Dadas-Stasiak E, Jung A, Jobs K, et al. An assessment of fractional exhaled nitric oxide in children with allergic rhinitis [J]. Pediatrics \& Family Medicine, 2017, 12(3):285-295.

[10] Han D H. Clinical application of fractional exhaled nitric oxide in pediatric allergic rhinitis [J]. 2015, 3(6):385.

[11] Giovannelli J, Chérotkornobis N, Hulo S, et al. Both exhaled nitric oxide and blood eosinophil count were associated with mild allergic asthma only in non-smokers. [J]. Clinical \& Experimental Allergy Journal of the British Society for Allergy \& Clinical Immunology, 2015, 46(4):543-554.

[12] Zhu Z, Xie Y, Guan W, et al. Effects of leukotriene D4 nasal challenge on bronchial responsiveness and inflammation in asthmatic patients with allergic rhinitis. [J]. Journal of Thoracic Disease, 2017, 9(2):271. 